\title{
Nephrotic syndrome in a patient with metastatic melanoma: beyond the obvious
}

\author{
Ana Messias ${ }^{1}$, Joaquim Calado ${ }^{1,2}$, Helena Viana ${ }^{1,3}$, Fernando Nolasco ${ }^{1}$ \\ ${ }^{1}$ Nephrology Department, Hospital Curry Cabral, Centro Hospitalar Universitário de Lisboa Central. \\ ${ }^{2}$ Centre for Toxicogenomics and Human Health (ToxOmics), Genetics, Oncology and Human Toxicology, Nova Medical School - Universidade Nova de Lisboa. \\ ${ }^{3}$ Laboratory of Renal Morphology, Nephrology Department, Hospital Curry Cabral, Centro Hospitalar Universitário de Lisboa Central.
}

\section{ABSTRACT}

Nephrotic syndrome in a patient with metastatic melanoma can occur in the context of a paraneoplastic glomerulopathy or as a complication of treatment. New oncologic immunotherapies, including immune checkpoint inhibitors, have been frequently associated with interstitial nephritis and, in rare cases, with nephrotic syndrome. This article presents the case of a 52-year-old man diagnosed with metastatic melanoma who was admitted with acute kidney injury and nephrotic syndrome after having started immune checkpoint inhibitors. After investigation, acute kidney injury was attributed to immunotherapy-associated acute interstitial nephritis, and nephrotic syndrome was found to be the result of $A A$ amyloidosis, which is a rare complication of metastatic melanoma.

Key-Words: amyloidosis, immunotherapy, melanoma, nephritis

\section{INTRODUCTION}

The approach to a patient with malignant tumor and kidney disease is complex since the disease etiology can have multiple causes. In addition to paraneoplastic glomerulopathies or direct kidney invasion by tumor cells, oncologic drug nephrotoxicity plays an important role in renal damage.

In the setting of nephrotic syndrome and malignant tumor, membranous glomerulopathy is the most common diagnosis ${ }^{1}$, which can be the first manifestation of tumor ${ }^{2}$. Nephrotic syndrome can also occur as an iatrogenic complication of cancer immunotherapy, including interferon therapy ${ }^{3}$. More recently, there have been case reports of nephrotic syndrome caused by immune-checkpoint inhibitors (ICPIs), mainly in the setting of minimal change disease ${ }^{4,5,6}$

ICPI therapy is an immunotherapeutic strategy which, instead of targeting tumor cells directly as chemotherapy, interferes with of cancer cells' ability to avoid an attack by the immune system, reinvigorating pre-existing anticancer immune responses ${ }^{7}$. ICPIs inhibit intrinsic down-regulators of T cells, such as cytotoxic T-lymphocyte-associated protein 4 (CTLA-4) and programmed cell death protein-1 pathway (PD-1) or its ligand (PD-L1) ${ }^{8}$. In the last decade, they have been approved by the US Food and Drug Administration (FDA) for the treatment of several malignant tumors, with promising outcomes ${ }^{9}$.

Although their tolerance seems to be better than chemotherapy ${ }^{10}$, ICPIs can cause immune-related adverse events (irAEs) in off-target organs, via several mechanisms which remain to be elucidated ${ }^{11}$. More than $50 \%$ of patients develop an irAE, which is severe or life-threatening in approximately $20 \%$ of cases $^{12}$. Skin, gastrointestinal tract and endocrine glands are most commonly involved ${ }^{9}$. Nephrotoxicity is a rare complication, with acute kidney injury (AKI) due to acute interstitial nephritis (AIN) the most commonly documented lesion ${ }^{13,14}$. Its incidence has been reported as low as $1.4 \%$ with monotherapy and up to $4.9 \%$ when combination therapy has been used ${ }^{15}$. The incidence of grade III and IV AKI (defined as the need for hospitalization or for urgent dialysis, respectively, according to National Cancer Institute Common Terminology Criteria for Adverse Events ${ }^{16}$ ) was $0.6 \%{ }^{15}$.

Renal biopsy plays an important role in identifying the underlying kidney disease in the setting of malignant tumor, which is essential for therapeutic decision and prognosis.

The authors report a case of a 52-year-old caucasian male patient displaying metastatic melanoma and kidney disease which manifested as AKI and nephrotic syndrome.

\section{CASE REPORT}

A 52-year-old man with an history of malignant melanoma diagnosed over 20 years ago, initially treated with surgery and chemotherapy, has been regularly followed by an oncologist. In the last 2 years, lung and lymph nodes metastatic involvement were identified and the patient was submitted to radiotherapy, chemotherapy and immunotherapy with ipilimumab (an intravenous monoclonal antibody against CTLA- 4 administered at $3 \mathrm{mg} / \mathrm{Kg}$ every 3 weeks until completing 4 cycles). Unfortunately, disease control was poor with subsequent positron-emission tomography diagnosis of tumor invasion of liver and bones four months later. After one year with rescue therapy with nivolumab (an intravenous anti-PD-1 monoclonal antibody 
administered at $240 \mathrm{mg}$ every 2 weeks), the patient was referred to our nephrology clinic for the evaluation of AKI [serum creatinine (Scr) rising from 0.9 to $2.2 \mathrm{mg} / \mathrm{dL}$ [normal range (NR) $0.72-1.25 \mathrm{mg} / \mathrm{dL}$ ] between February and April]. Additional significant medical history included lisinopril therapy for systemic hypertension (diagnosis 5 years ago; no history of vascular complications) and diagnosis of kidney stones with acute colic episode 4 years ago. In the last few months, along with omeprazole, the patient was receiving celecoxib therapy to control rib pain and has recently been submitted to radiotherapy to limit rib metastatic injury. On presentation, blood pressure was $115 / 80 \mathrm{mmHg}$, pulse rate 75 beats/min and his physical examination

\section{Figure 1}

PAS (Periodic Acid-Schiff), $\times 200$

Glomerular and vascular amorphous material deposits.

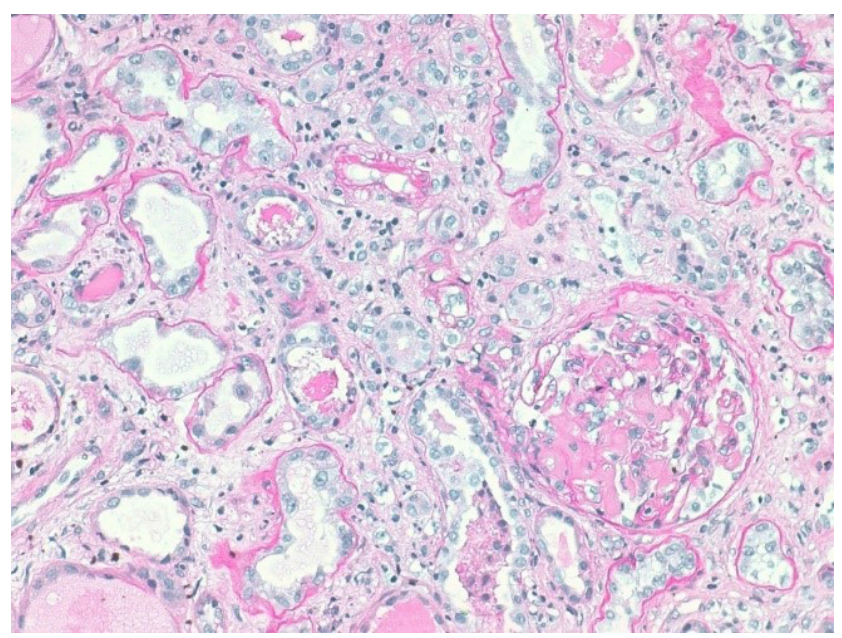

\section{Figure 2}

Congo red, $x 200$

Amorphous material deposits were identified as amyloid by Congo red staining

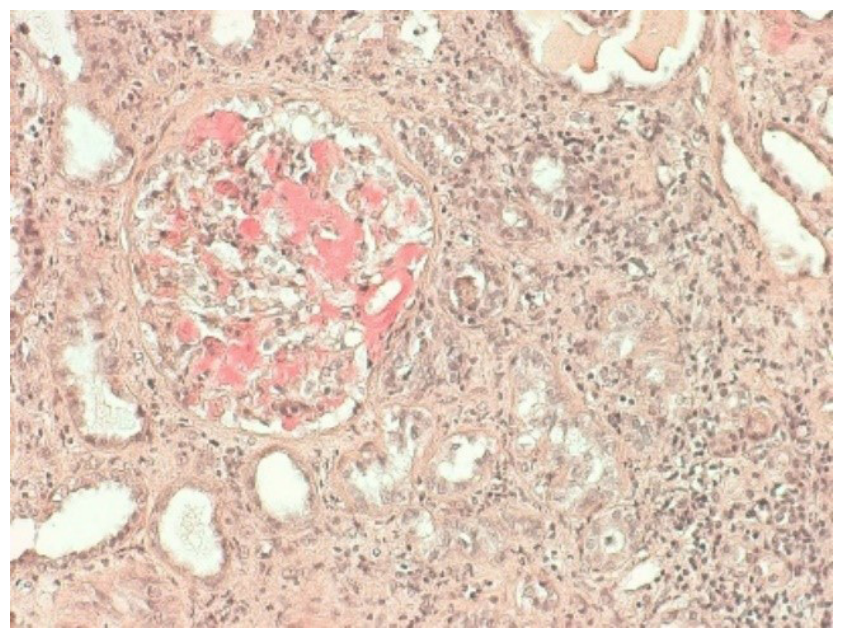

revealed a bilateral lower-extremity oedema, without fever, skin rash or pruritus. Chest auscultation and abdomen palpation were normal. Laboratory tests demonstrated normocytic normochromic anemia (hemoglobin $10.2 \mathrm{~g} / \mathrm{dL}$, NR $13-17 \mathrm{~g} / \mathrm{dL}$ ), Scr $4.4 \mathrm{mg} / \mathrm{dL}$, without electrolyte or acid-base disturbances; normal hepatic function, hypoalbuminemia (serum albumin $2.97 \mathrm{~g} / \mathrm{dL}, \mathrm{NR} 3.2-4.6 \mathrm{~g} / \mathrm{dL}$ ), hypercholesterolemia [total serum cholesterol $305 \mathrm{mg} / \mathrm{dL}$ ( $\mathrm{NR}<190 \mathrm{mg} / \mathrm{dL}$ ) and triglycerides $467 \mathrm{mg} / \mathrm{dL}(\mathrm{NR}<150 \mathrm{mg} / \mathrm{dL})]$ and nephrotic range proteinuria (a urine protein-to-creatinine (UPC) ratio of $20561 \mathrm{mg} / \mathrm{g}$, $\mathrm{NR} \leq 200 \mathrm{mg} / \mathrm{g}$ ). In addition to proteinuria, urinalysis showed sterile pyuria with 23 white blood cells per cubic millimeter $\left(\mathrm{mm}^{3}\right)(\mathrm{NR}<11$

\section{Figure 3a}

Immunohistochemistry, x400

Acute tubulointerstitial nephritis with infiltration of T-lymphocytes (CD3 staining).

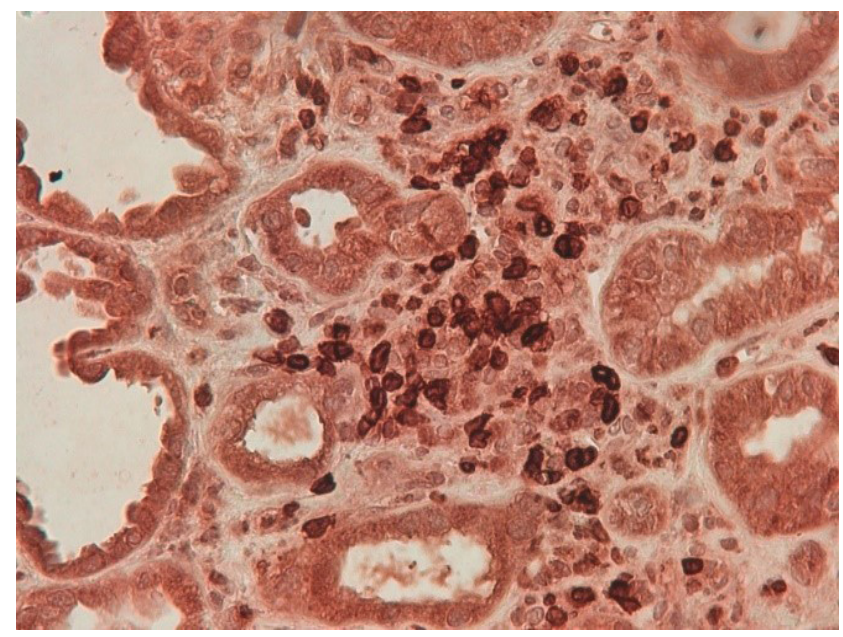

\section{Figure 3b}

Immunohistochemistry, x400

Acute tubulointerstitial nephritis with infiltration of T-lymphocytes (CD8 staining).

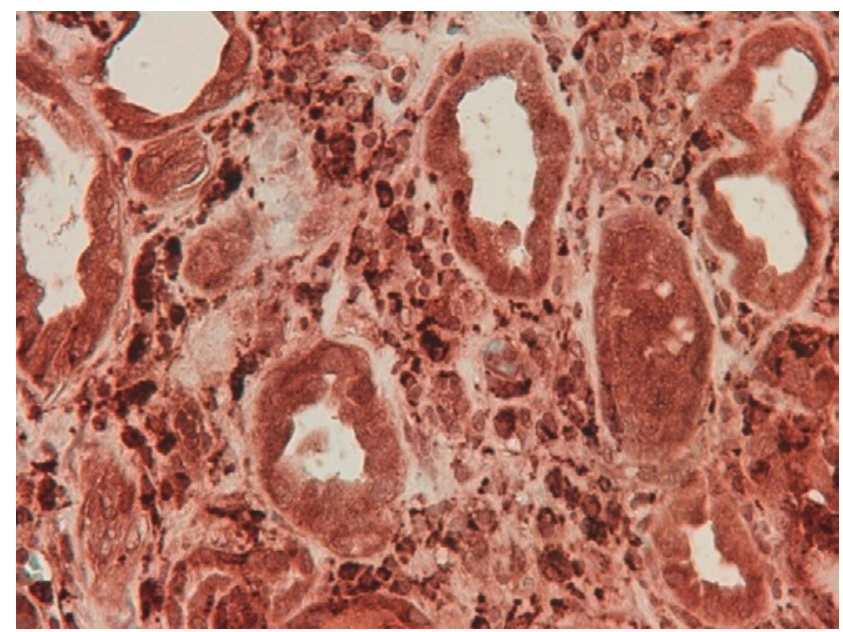


$\mathrm{WBC} / \mathrm{mm}^{3}$ ), without microscopic hematuria (4 red blood cells $/ \mathrm{mm}^{3}$, $\mathrm{NR}<17 \mathrm{RBC} / \mathrm{mm}^{3}$ ). No serum or urinary eosinophilia was found or tested, respectively. Screening for hepatitis $B$ and $C$ viruses and human immunodeficiency virus was negative. Immunological tests (antinuclear and anti-double stranded DNA antibodies, rheumatoid factor, anti-neutrophil cytoplasmic autoantibodies, anti-glomerular antibodies) were also negative. Complement levels and serum immunoglobin levels were within the normal range. Electrophoresis and immunofixation did not identify the presence of a monoclonal immunoglobin component in the serum. Renal ultrasonography showed a size asymmetry (168 $\mathrm{mm}$ for the left and $98 \mathrm{~mm}$ for the right kidney) and a moderate increase of parenchymal echogenicity with normal cortex thickness, without evidence of hydronephrosis. Renal vein thrombosis was excluded by doppler ultrasound. Nivolumab and other nephrotoxic agents (celecoxib and omeprazole) were stopped and oral prednisolone $(60 \mathrm{mg} /$ day, at $1 \mathrm{mg} / \mathrm{Kg} /$ day) was initiated by the oncological medical team, without clinical or renal function improvement. After 4 weeks of prednisolone ( $40 \mathrm{mg} /$ day in the last week), our nephrology department was consulted, performing a renal biopsy. Light microscopy demonstrated 11 glomeruli, one of which was globally sclerosed, and some medium caliber vessels. The remaining glomeruli, as well as vessel walls, contained pale, amorphous and eosinophilic deposits (Figure 1) identified as amyloid by Congo red staining (Figure 2) (polarized light not available). Severe AIN with interstitial oedema and infiltration of mono and polymorphic inflammatory cells was found. The interstitial inflammatory infiltrate was predominantly composed of cytotoxic T lymphocytes (CD3+, CD8+) and macrophages (CD68+), with scanty $B$ cells (CD20+) and plasma cells (CD138+) (Figures 3a and $3 b)$. Extensive necrosis of tubular cells in association with tubular inflammatory infiltrate was recognized and no interstitial granulomas were present. Immunofluorescence revealed glomerular and vascular staining for amyloid A protein (Figure 4) but no staining for kappa or lambda light chains. Electron microscopy was not performed. These findings were compatible with AA amyloidosis and acute tubulointerstitial nephritis. The patient was treated with an increased dose of

\section{Figure 4}

Immunofluorescence, $x 400$

Presence of amyloid A protein in glomeruli and arterioles.

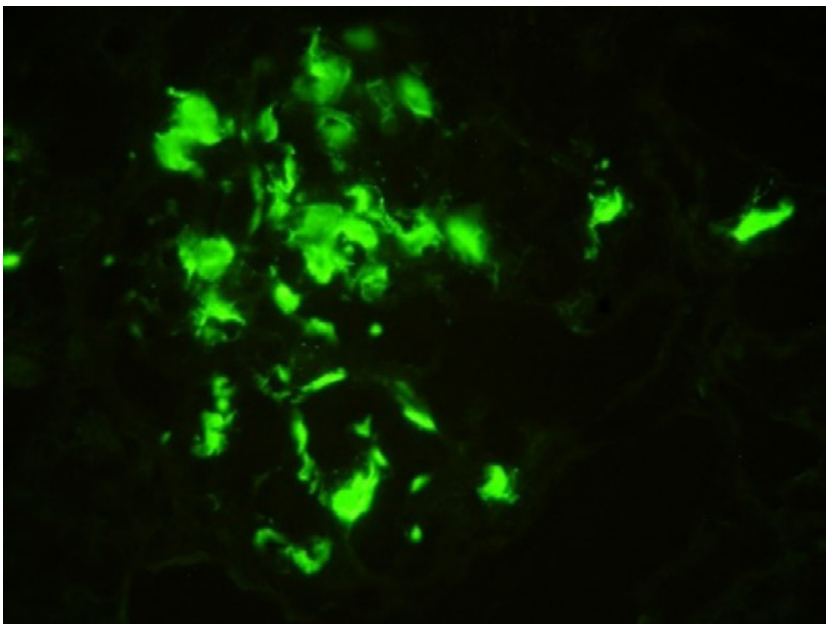

prednisolone (60 mg daily, at $1 \mathrm{mg} / \mathrm{Kg} / \mathrm{day}$ ) for 4 weeks, without recovery of kidney function or remitance of proteinuria, needing maintenance haemodialysis 3 times per week and dying two months later due to progression of his underlying cancer.

\section{DISCUSSION}

We report a case of a patient with metastatic melanoma under ICPI therapy who presented with AKI and nephrotic syndrome. ICPI therapy was presumed to be the cause of both AKI and nephrotic syndrome. Renal biopsy was performed showing AIN and AA amyloidosis. Thus, while AKI may have occurred in the context of AIN-associated ICPI, nephrotic syndrome was likely related to paraneoplastic AA amyloidosis.

Melanoma is the most aggressive type of skin cancer with remarkable propensity for metastatic spreading and is often refractory to anticancer drugs ${ }^{8}$. Nephrotic syndrome can be developed when there is melanoma associated with membranous nephropathy, and is the first manifestation of melanoma in some rare cases ${ }^{17}$. Although there is data demonstrating that serum amyloid $A$ (SAA) protein is intensively expressed by malignant melanoma cells and could be a marker for predicting a high risk of tumor progression ${ }^{18}, \mathrm{AA}$ amyloidosis is an unusual complication of melanoma. A successful treatment of the underlying process can lead to the stabilization or improvement of renal function, reduction in protein excretion, and partial resolution of amyloid deposits ${ }^{19}$. Cancer cells are known to produce SAA protein ${ }^{20}$ although AA amyloidosis is rarely associated with solid organ malignancies, metastatic disease and Hodgkin's lymphoma $(<7 \%)^{21,22}$.

Significant treatment advances for unresectable or metastatic melanoma have occurred in the past few years, with a notable impact on prognosis ${ }^{23}$. Ipilimumab (anti-CTLA-4) and nivolumab (anti-PD-1) were the first ICPIS FDA-authorized for the treatment of advanced malignant melanoma. ${ }^{24,25}$ AIN has been described as the most common kidney lesion related to ICPI therapy and there have been case reports of AIN caused by ipilimumab and nivolumab treatment ${ }^{15,26-31}$. Clinical and histologic features of ICPI-induced AIN are similar to other causes of $\mathrm{AIN}^{15}$. ICPI-associated AKI has been more frequently reported to occur late after drug initiation (after several months), unlike classic drug-induced AKI, suggesting an alternative mechanism ${ }^{15}$. However, the pathophysiology of renal toxicity is still poorly understood and some cases with toxicity occurring after only 21 days of drug initiation ${ }^{15}$ have been reported. Currently the mechanism proposed purports that the delay which is seen between drug initiation and AKI development reflects the immunological process of tolerance loss against endogenous kidney antigens whose exposure is induced by ICPI therapy ${ }^{32}$. It is important to exclude the possibility that AIN was caused by a concomitant medication with known association with AIN rather than an ICPI. A proton-pump inhibitors (omeprazole) and a nonsteroidal anti-inflammatory drug (celecoxib) were started by our patient two months prior of AKI development, making a causal relationship less likely ${ }^{33}$.

Nephrotic syndrome can be associated with ICPI therapy ${ }^{4}$, with only one case of AA amyloidosis as a side-effect described in literature $^{31}$. This patient had partial renal function recovery after 
pembrolizumab (anti-PD 1) discontinuation and steroid treatment (methylprednisolone $1 \mathrm{mg} / \mathrm{Kg})^{31}$. In this case, infliximab (anti-TNF alfa) was also used, due to refractory colitis which can also contribute to renal recovery ${ }^{31}$. Infliximab or other oncologic therapies were not used in our patient.

These multiple possible diagnoses highlight the requirement of renal biopsy for patients with kidney dysfunction in the setting of cancer and ICPI therapy, because a misleading diagnosis may lead to undue therapy discontinuation and high-dose steroid regimen, which carries additional risks and delays the start of appropriate treatment.

In our case, renal biopsy was important to confirm diagnosis. Firstly, we assumed that the clinical picture could be fully explained by nivolumab treatment. Cessation of ICPI and steroid therapy did not result in renal function recovery. Alternative therapies, such as infliximab and mycophenolate mofetil, have been described, but data pertaining to their use is too scarce ${ }^{11}$ and therefore we have decided to perform a renal biopsy. Histology results showed not only AIN, secondary to immunotherapy, but also AA amyloidosis that was attributed to be a complication of metastatic melanoma, rather than ICPI therapy. In most patients with malignancy-associated AA amyloidosis, the course of proteinuria typically parallels that of the underlying malignancy, with remission of nephrotic syndrome following successful malignancy treatment ${ }^{2}$. This is congruent with our patient, whose proteinuria continued to worsen and who ultimately died due to melanoma progression.

We emphasize the utility of a urinalysis before the initiation of PD-1 inhibitor, which unfortunately was not available in our patient. The presence of proteinuria in a prior urinalysis would have reinforced the diagnosis of AA amyloidosis associated with melanoma. The lack of such important pre-treatment clinical information reinforces the common dilemma in clinical practice of differentiating kidney injury of paraneoplastic cause from cancer treatment toxicity.

\section{CONCLUSION}

Given the increasing use of immunotherapies, nephrologists should be aware of their potential immune-related nephrotoxicity. In the setting of malignant tumor and ICPI therapy, patients with urinalysis and kidney function assessment should be closely monitored, to allow for rapid recognition of kidney injury and early intervention.

\section{Disclosure of potential conflicts of interest: none declared}

\section{References}

1. Bacchetta J, Juillard L, Cochat P, Droz JP. Paraneoplastic glomerular diseases and malignancies Crit Rev Oncol Hematol. 2009 Apr;70(1):39-58

2. Lefaucheur C, Stengel B, Nochy D. Membranous nephropathy and cancer: epidemiologic evidence and determinants of high-risk cancer association. Kidney Int. 2006;70:1510-1517

3. Markowitz GS, Nasr SH, Stokes MB, D’Agati VD. Treatment with IFN-\{alpha\}, -\{beta\}, or -\{gamma\} is associated with collapsing focal segmental glomerulosclerosis. Clin J Am Soc Nephrol. 2010 Apr;5(4):607-615
4. Kitchlu A, Fingrut W, Avila-Casado C et al. Nephrotic syndrome with cancer immunotherapies: A report of 2 cases. Am J Kidney Dis. 2017 Oct;70(4):581-585

5. Kidd JM, Gizaw AB. Ipilimumab-associated minimal-change disease. Kidney Int. 2016 Mar;89(3):720

6. Bickel A, Koneth I, Enzler-Tschudy A, Neuweiler J, Flatz L, Früh M. Pembrolizumab-associated minimal change disease in a patient with malignant pleural mesothelioma. BMC Cancer. 2016 Aug 19;16:656

7. Chen DS, Mellman I. Oncology meets immunology: the cancer-immunity cycle. Immunity. 2013 Jul 25;39(1):1-10

8. Mattia G, Puglisi R, Ascione B, Malorni W, Carè A, Matarrese P. Cell death-based treatments of melanoma: conventional treatments and new therapeutic strategies. Cell Death Dis. 2018 Jan 25;9(2):112

9. Postow MA, Sidlow R, Hellmann MD. Immune-related adverse events associated with immune checkpoint blockade. N Engl J Med. 2018 Jan 11;378(2):158-168

10. Lemiale V, Meert AP, Vincent F et al. Severe toxicity from checkpoint protein inhibitors: what intensive care physicians need to know? Ann Intensive Care. 2019 Feb 1;9(1):25

11. Shingarev R, Glezerman IG. Kidney complications of immune checkpoint inhibitors: a review. Am J Kidney Dis. 2019 Oct; 74(4):529-537

12. Perazella MA, Sprangers B. AKI in patients receiving immune checkpoint inhibitors. Clin J Am Soc Nephrol. 2019 Jul 5;14(7):1077-1079

13. Sury K, Perazella MA, Shirali AC. Cardiorenal complications of immune checkpoint inhibitors. Nat Rev Nephrol 2018 Sep;14(9):571-588

14. Perazella MA, Shirali AC. Nephrotoxicity of cancer immunotherapies: past, Present and Future. J Am Soc Nephrol. 2018 Aug;29(8):2039-2052

15. Cortazar FB, Marrone KA, Troxell ML et al. Clinicopathological features of acute kidney injury associated with immune checkpoint inhibitors. Kidney Int. 2016 Sep;90(3):638-647

16. Common Terminology Criteria for Adverse Events (CTCAE) Version 5.0. National Institutes of Health, National Cancer Institute, US Department of Health and Human Services; 2017.

17. Faria TV, Baptista MAF, Burdman EA, Cury P. Glomerular deposition of imune complexes as a first manifestation of malignant melanoma - a case report. Renal Failure 2010. 32(10):1223-1225

18. Findeisen $P$, Zapatka $M$, Peccerella $T$ et al. Serum amyloid $A$ as a prognostic marker in melanoma identified by proteomic profiling. J Clin Oncol. 2009 May 1;27(13):2199-2208

19. Babu A, Lachmann H, Pickett T, Boddana P, Ludeman L. Renal cell carcinoma presenting as AA amyloidosis: a case report and review of the literature. CEN Case Rep. 2014 May;3(1):68-74

20. Moshkovskii SA. Why do cancer cells produce serum amyloid A acute-phase protein? Biochemistry. 2012 Apr;77(4):339-341

21. Dictor M, Hasserius R. Systemic amyloidosis and non-hematologic malignancy in a large autopsy series. Acta Pathol Microbiol Scand A. 1981 Nov;89(6):411-416

22. De Asúa DR, Costa R, Galván JM, Filigheddu MT, Trujillo D, Cadiñanos J. Systemic AA amyloidosis: epidemiology, diagnosis, and management. Clin Epidemiol. 2014; 6:369-377

23. Lugowska I, Teterycz P, Rutkowski P. Immunotherapy of melanoma. Contemp Oncol (Pozn). 2018 Mar; 22(1A):61-67

24. National Institute for Health and Care Excellence. Ipilimumab for previously untreated advanced (unresectable or metastatic) melanoma. Available at https://www.nice.org.uk/guidance/ta319. Accessed June 15, 2019

25. National Institute for Health and Care Excellence. Nivolumab in combination with ipilimumab for treating advanced melanoma. Available from: https://www.nice.org.uk/guidance/ta400. Accessed June 15, 2019

26. Voskens $\mathrm{CJ}$, Goldinger SM, Loquai $\mathrm{C}$ et al. The price of tumor control: an analysis of rare side effects of anti-CTLA4 therapy in metastatic melanoma from the ipilimumab network. PLoS One. 2013;8(1):e53745

27. Izzedine $\mathrm{H}$, Gueutin V, Gharbi $\mathrm{C}$ et al. Kidney injuries related to ipilimumab. Invest New Drugs. 2014 Aug;32(4):769-773

28. Thajudeen B, Madhrira M, Bracamonte E, Cranmer LD. Ipilimumab granulomatous interstitial nephritis. Am J Ther. 2015 May-Jun;22(3):e84-7

29. Murakami N, Borges TJ, Yamashita M, Riella LV. Severe acute interstitial nephritis after combination immune-checkpoint inhibitor therapy for metastatic melanoma. Clin Kidney J. 2016 Jun;9(3):411-417

30. Bottlaender L, Breton AL, de Laforcade L, Dijoud F, Thomas L, Dalle S. Acute interstitial nephritis after sequential ipilumumab - nivolumab therapy of metastatic melanoma. J Immunother Cancer. 2017 Jul 18;5(1):57

31. Mamlouk O, Selamet U, Machado S et al. Nephrotoxicity of immune checkpoint inhibitors beyond tubulointerstitial nephritis: single-center experience. J Immunother Cancer. 2019 Jan 6;7(1):2

32. Shirali AC, Perazella MA, Gettinger S. Association of acute interstitial nephritis with programmed cell death 1 inhibitor therapy in lung cancer patients. Am J Kidney Dis. 2016 Aug;68(2):287-291

33. Zhang X1, Donnan PT, Bell S, Guthrie B. Non-steroidal anti-inflammatory drug induced acute kidney injury in the community dwelling general population and people with chronic kidney disease: systematic review and meta-analysis. BMC Nephrol. 2017 Aug 1;18(1):256

\section{Correspondence to:}

Ana Messias, MD.

Department of Nephrology, Centro Hospitalar Universitário de Lisboa Central,

Rua da Beneficência 8, 1069-166 Lisbon, Portugal.

E-mail: anamsmessias@gmail.com 